\title{
Argon- and nitrogen-plasma pulses decrease facial sebum excretion in oily human skin by regulating lipogenesis and proliferation in human sebocytes
}

\section{Sung Bin Cho}

Yonsei Seran Dermatology and Laser Clinic, Seoul, Korea

\section{Seung-Ju Lee}

Yonsei University College of Medicine

Dae San Yoo

Yonsei University College of Medicine

\section{Song-Ee Kim}

Yonsei University College of Medicine

\section{Christos C. Zouboulis}

Brandenburg Medical School Theodor Fontane

\section{Sang Eun Lee ( $\nabla$ jennifer823@yuhs.ac)}

Yonsei University College of Medicine

\section{Research Article}

Keywords: plasma, argon, nitrogen, atmospheric-pressure plasma, non-thermal plasma, hyperseborrhea, sebocytes

Posted Date: August 4th, 2021

DOl: https://doi.org/10.21203/rs.3.rs-729953/v1

License: (c) (i) This work is licensed under a Creative Commons Attribution 4.0 International License. Read Full License 


\section{Abstract}

Although several energy devices targeting sebaceous glands have been developed, an effective and safe therapeutic tool for hyperseborrhea is needed. Non-thermal atmospheric-pressure plasma (NTAPP) induces microscopic tissue reactions in sebaceous glands of rat skin in vivo. Fifteen healthy volunteers with oily facial skin underwent three sessions of argon- and nitrogen-NTAPP treatment. The casual sebum level, sebum excretion rate, porphyrin index, and histological analysis were evaluated. Argon- and nitrogen-NTAPP pulses were applied to testosterone and linoleic acid (T/LA)-treated human SZ95 sebocytes. Lipids were assessed using BODIPY and quantitative Nile red staining. Proliferation and differentiation markers were assessed. Argon- and nitrogen-NTAPP pulses suppressed casual sebum levels and sebum excretion rates in the skin, at week 4 . The porphyrin index revealed a $38 \%$ reduction at week 2. Skin biopsy samples showed no obvious thermal coagulation, but the number of $\mathrm{Ki} 7^{+}$cells in the sebaceous glands decreased at week 2. Argon- and nitrogen-NTAPP inhibited T/LA-induced increases in lipid synthesis, $\mathrm{Ki} 67^{+}$cells, and peroxisome proliferator-activated receptor- $\nabla$ transcription in human sebocytes without apoptosis. Argon- and nitrogen-NTAPP therapy is a safe and effective method for reducing sebum excretion in oily human skin and inhibits lipogenesis and cell proliferation in human sebocytes.

\section{Background}

Plasma consists of charged particles of ions and electrons, neutral atoms, photons, and radicals, which can be created artificially by delivering energy to an inert gaseous source1,2. Artificially created plasma is classified as thermal or non-thermal plasma based on temperature, and low- or atmospheric-pressure plasma based on gas pressure2. Among these, non-thermal atmospheric-pressure plasma (NTAPP) is used to treat various medical disorders, including acute and chronic wounds, inflammatory disorders, and infectious diseases, without inducing direct thermal damage to living tissues1-8. Several inert gaseous sources, particularly ambient air, helium, argon, and nitrogen, are used to generate NTAPP for medical purposes9-11. Therein, ultra-high-frequency radiofrequency or microwave energy is generally used to generate excited ionized gas9,11. A pilot study histologically analyzed argon- and nitrogen-NTAPPinduced immediate and late rat skin reactions, and reported that tissue reactions varied depending on the gaseous source of different emission spectra (argon versus nitrogen) and the treatment settings 12 . NTAPP treatment induced microscopic tissue reactions in the follicular epithelium and sebaceous glands12. Interestingly, argon-NTAPP-induced vacuolar tissue reactions were observed in the epidermis, which propagated along the follicular epithelium to the entrance of the sebaceous duct12. Histological features of thermal modification and coagulation were observed in the sebocytes, but not in the surrounding dermal stromal tissue, of argon-NTAPP-treated rat skin12. Low-energy nitrogen-NTAPP treatments also elicited thermal tissue reactions in the sebaceous glands, while preserving the surrounding dermal stromal tissue, whereas, high-energy nitrogen-NTAPP delivery resulted in extensive thermal coagulation of the epidermis and upper-to-mid dermis12. These findings suggest the effect of NTAPP on the human sebaceous gland physiology; however, supporting data are lacking. Sebum is 
important for maintaining stratum corneum hydration, low skin surface $\mathrm{pH}$, and antimicrobial barrier function of the skin. However, excess sebum production can contribute to the development of acne. Therefore, we investigated the effect of argon- and nitrogen-NTAPP on sebum production, differentiation, and proliferation of sebaceous gland cells in oily human skin in vivo and in cultured human sebocytes.

\section{Results}

\section{Argon- and nitrogen-NTAPP pulses reduced facial sebum excretion in oily human skin}

First, we assessed the effect of argon- and nitrogen-NTAPP pulses on sebum excretion in subjects with oily facial skin. Fifteen healthy volunteers aged 19-35 years were enrolled in this trial. Table 1 shows the demographic and baseline characteristics. At baseline, the mean $( \pm S D)$ casual sebum levels on the forehead and both cheeks were $238.2 \pm 58.18$ and $191.7 \pm 51.66 \mathrm{mg} / \mathrm{cm}^{2}$, respectively. 
Table 1

Subject demographics.

\begin{tabular}{|ll|}
\hline Characteristics & Value $(\boldsymbol{n}=15)$ \\
\hline Age, years & \\
\hline Mean \pm standard deviation & $28.1 \pm 5.2$ \\
\hline Range & $19-35$ \\
\hline Sex, $n(\%)$ & $8(53.3)$ \\
\hline Male & $7(46.7)$ \\
\hline Female & $238.2 \pm 58.18$ \\
\hline Sebumeter values (mg/cm $\left.{ }^{2}\right)^{\text {a }}$ & $191.7 \pm 51.66$ \\
Forehead & \\
Both cheeks & \\
Fitzpatrick's skin type, $n(\%)$ & 0 \\
\hline I & 0 \\
\hline II & $15(100)$ \\
\hline III & 0 \\
\hline IV & 0 \\
\hline V & \\
\hline Korean acne grading system, $n(\%)$ & \\
\hline 1 & \\
\hline aData are presented as mean \pm standard deviation. & \\
\hline
\end{tabular}

The casual sebum levels on the forehead and both cheeks decreased significantly compared with baseline at 2 and 4 weeks after treatment, with a maximal reduction observed at week 4 . The mean casual sebum levels measured on the forehead, left cheek, and right cheek decreased by $16.0,19.4$, and $18.7 \%$, respectively, at week 2 , and by 28,24 , and $22.3 \%$, respectively, at week 4 compared to baseline. The sebosuppressive effect of NTAPP gradually attenuated over time to week 8 (Fig. 1a). The rates of sebum excretion on the forehead, left cheek, and right cheek also decreased by $12.4,12.7$, and $18.2 \%$, respectively, at week 2 , and by $26.6,22.7$, and $23 \%$, respectively, at week 4 compared to baseline and then the inhibitory effects attenuated by week 8 (Fig. 1b). Most subjects experienced temporary erythema, which spontaneously resolved within $24 \mathrm{~h}$. No treatment-related severe adverse events were observed during the 8-week follow-up period. 


\section{Porphyrin fluorescence in the seborrheic area decreased following argon- and nitrogen-NTAPP pulse treatment}

Cutibacterium acnes (C. acnes) occurs predominantly in the sebaceous gland-rich areas of the skin. To examine the effect of argon- and nitrogen-NTAPP pulses on the abundance of $C$. acnes on the seborrheic area of the oily human skin, porphyrins contents were analyzed in seven subjects at baseline and week 2 using digital ultraviolet (UV) photographs with an image analyzer. UV-induced red fluorescence in the follicle indicates the presence of porphyrins, including coproporphyrin III and protoporphyrin IX, which are produced by anaerobic Gram-negative bacteria, such as $C$. acnes ${ }^{13,14}$. The average porphyrin index decreased on the forehead and both cheeks by $27.7 \%$ and $37.7 \%$, respectively, compared to baseline at week 2 (Fig. 2).

Argon- and nitrogen-NTAPP pulses reduced proliferation marker Ki67 in the basal sebaceous gland cells and epithelial cells in sebaceous ducts of oily human skin

To investigate the effect of NTAPP pulses on the histological changes of the human sebaceous glands, skin biopsies were obtained from the zygomatic area of two volunteers at baseline and week 2. Biopsies taken 2 weeks after treatment showed no apparent coagulation in the sebaceous glands and surrounding dermis. Although hematoxylin-eosin staining did not reveal obvious changes in the number or size of sebaceous glands, the number of $\mathrm{Ki}^{+} 7^{+}$cells in the basal layer of sebaceous glands and in the epithelium of sebaceous ducts significantly decreased at week 2 compared to baseline, as determined by immunohistochemistry (Fig. 3).

There were no significant changes in the nuclear peroxisome proliferator-activated receptor- $($ PPARY)stained sebocytes between baseline and week 2 (data not shown). Nonequilibrium atmospheric-pressure plasma and plasma-activated medium have been shown to induce the intracellular accumulation of reactive oxygen species (ROS) ${ }^{15}$; therefore, we also evaluated a biomarker of oxidative stress, 4Hydroxynonenal (4-HNE), and found no significant changes in its immunoreactivity between baseline and week 2 (data not shown).

\section{Argon- and nitrogen-NTAPP pulses suppressed lipid production in cultured human SZ95 sebocytes}

To elucidate the mechanisms involved in the sebosuppressive effects of argon- and nitrogen-NTAPP pulses in oily human skin in vivo, we evaluated the effects of NTAPP pulses on the lipogenesis of human SZ95 sebocytes, which possess the major characteristics of normal sebocytes ${ }^{16}$. First, we examined the cytotoxicity of argon- and nitrogen-NTAPP pulses in sebocytes using the MTT assay. The 20 or 60 pulses of argon NTAPP at $0.75 \mathrm{~J}$, and 5 or 10 pulses of nitrogen NTAPP at $1.5 \mathrm{~J}$ did not induce detectable changes in sebocyte viability over $48 \mathrm{~h}$ (Fig. 4a), thus these experimental settings were used in subsequent experiments. To investigate the effect of NTAPP on pro-acne agents-induced lipogenesis in sebocytes, we treated argon- or nitrogen-NTAPP pulses to SZ95 sebocytes immediately or $24 \mathrm{~h}$ after 
treatment with a combination of testosterone and linoleic acid (T/LA) and then the intracellular neutral lipid contents were assessed by BODIPY and quantitative Nile red staining. 20 or 60 pulses of argonNTAPP at $0.75 \mathrm{~J}$, immediately or $24 \mathrm{~h}$ after the treatment with T/LA, significantly suppressed T/LAenhanced lipid accumulation in sebocytes as assessed by BODIPY staining (Fig. $4 \mathrm{~b}$ and $\mathrm{c}$ ) and quantitative Nile red staining (Fig. 4d). Further, 5 or 10 pulses of nitrogen-NTAPP pulses at $1.5 \mathrm{~J}$ also suppressed T/LA-induced neutral lipid synthesis in sebocytes when applied immediately or $24 \mathrm{~h}$ after T/LA treatment (Fig. 4b-d). To elucidate the molecular mechanism by which argon- and nitrogen-NTAPP pulses suppressed sebaceous lipid synthesis, we investigated the effect of argon- and nitrogen-NTAPP pulses on the expression of PPARY, a potential modulator of sebocyte differentiation and lipid synthesis. PPARY gene expression increased following exposure to T/LA for $48 \mathrm{~h}$ and co-treatment with argonNTAPP ( $0.75 \mathrm{~J}, 60$ pulses) or nitrogen-NTAPP ( $1.5 \mathrm{~J}, 10$ pulses) suppressed the T/LA-induced upregulation of PPARY mRNA (Fig. 4e). These results clearly show that argon- and nitrogen-NTAPP suppresses androgen and PPAR-stimulator-induced lipogenesis in sebocytes, and also suggest that inhibition of PPARY induction may partially contribute to the sebosuppressive effect of NTAPP.

\section{Argon- and nitrogen-NTAPP pulses inhibited proliferation of SZ95 sebocytes in vitro}

To corroborate our findings that argon- and nitrogen-NTAPP treatment decreased the proliferation marker Ki67 in sebaceous gland cells of oily human skin in vivo, we examined the effects of NTAPP on the proliferation of cultured SZ95 sebocytes in vitro by measuring Ki- $67^{+}$cells. As previously reported, T/LA enhanced the proliferation of SZ95 sebocytes. The 20 or 60 pulses of argon-NTAPP at $0.75 \mathrm{~J}$, or 5 or 10 pulses of nitrogen-NTAPP at $1.5 \mathrm{~J}$ significantly suppressed the T/LA-induced increased in $\mathrm{Ki}-67^{+}$cells when applied immediately or $24 \mathrm{~h}$ after T/LA treatment (Fig. $5 \mathrm{a}$ and b). Our data suggest that argon- and nitrogen-NTAPP pulses inhibit the proliferation of human sebocytes without inducing apoptosis.

\section{Discussion}

In the present study, we demonstrate that three sessions of argon- and nitrogen-NTAPP pulses, for three consecutive weeks, reduce sebum excretion in oily facial skin, with a maximal effect at week 4 in most subjects, accompanied by the reduction in Ki-67-positive proliferating cells within the sebaceous glands. To the best of our knowledge, this is the first report that study the effect of NTAPP pulses on human sebaceous glands in vivo.

A previous study, using a rat skin model, showed that argon plasma induced microscopic changes in sebaceous glands and associated follicular epithelium without causing excessive thermal injury, and nitrogen plasma elicited energy-dependent thermal coagulation in pilosebaceous units ${ }^{12}$. In contrast to the previous murine results, histological examination of argon- and nitrogen-NTAPP pulses-treated human skin revealed no apparent changes associated with thermal damage in the interfollicular epidermis and sebaceous glands in this study. This discrepancy may be attributed to differences in the energy settings. In rat skin, argon- and nitrogen-NTAPP pulses were delivered at $0.85 \mathrm{~J} /$ pulses and 3 $\mathrm{J} /$ pulses, respectively; however, human skin was treated with a lower energy of argon ( $0.8 \mathrm{~J} / \mathrm{pulses})$ - and 
nitrogen ( $0.75 \mathrm{~J} /$ pulses)-NTAPP pulses for safety reasons. In addition, the effect of NTAPP at the same energy setting might be overestimated in rat skin because human skin is less permeable than rat skin. Moreover, tissue changes in rat skin were examined serially on day 1, 5, and 7 after plasma treatment, but human skin tissues were examined 2 weeks after treatment; thus, the possibility of an early transient tissue reaction cannot be ruled out. Further time-course examination of the histological changes in human skin following NTAPP pulses at various energy settings will be needed for future clinical applications. Taken together, our findings suggest that argon- and nitrogen-NTAPP pulses suppress sebum secretion in oily human skin, likely through inhibiting ductal and sebaceous cell proliferation rather than thermal damage to the sebaceous glands.

Depending on the doses, NTAPP has been shown to promote or inhibit cell proliferation in many types of cells. The biological effects of plasma are thought to be related to the generation of ROS. High levels of ROS can cause oxidative stress, cell cycle arrest, and DNA damage. In this study, NTAPP treatment on oily human skin did not induce a detectable change in the expression of 4-HNE, a marker of oxidative stress in the treated glands. These results suggest that the inhibition of cell proliferation in the sebaceous glands by NTAPP pulses at parameters used in this study did not primarily rely on excessive ROS production. To further investigate the molecular mechanisms behind the sebosuppressive effect of argon- and nitrogenNTAPP pulses in vivo, we examined the effects of NTAPP pulses on human sebocyte functions, including lipogenesis, proliferation, and differentiation. Argon- and nitrogen-NTAPP pulses inhibited the T/LAenhanced neutral lipid synthesis of human sebocytes when treated in the undifferentiated or T/LAinduced early differentiated state. This suggests that argon- and nitrogen-NTAPP pulses can inhibit excessive lipid production induced by acne-promoting stimuli, such as testosterone and linoleic acid. Recent evidences suggest that NTAPP regulates cell proliferation and differentiation in multiple cell types. Sebocyte differentiation is regulated by a complex network of transcriptional factors, of which PPARY has an important role in sebocyte maturation, inducing the synthesis of sebaceous lipid. We found that treatment of SZ95 sebocytes with argon- and nitrogen-NTAPP pulses during the initial $24 \mathrm{~h}$ of T/LAinduced differentiation inhibited the induction of PPARY gene expression $48 \mathrm{~h}$ after T/LA treatment. These data suggest that argon- and nitrogen-plasma pulses may decrease differentiation-induced lipogenesis in human sebocytes via inhibiting PPARy signaling.

Systemically and locally increased androgen leads to an increase of sebocyte proliferation in patients with acne. We found that both argon- and nitrogen-NTAPP pulses, in an energy setting that does not induce cell apoptosis, significantly decreased Ki67 + proliferative cells in T/LA-treated sebocytes, which is consistent with the histological changes in the sebaceous glands of human skin treated with NTAPP. Taken together, antiproliferative effect of NTAPP on human sebocytes in vitro and in vivo may contribute to the reduction of sebum production.

Notably, argon- and nitrogen-NTAPP treatment significantly reduced porphyrin levels in oily facial skin. Given that porphyrins are produced by the lipophilic commensal bacterium $C$. acnes, these findings suggest a suppressive effect of NTAPP against $C$. acnes. Recent studies have reported the antimicrobial effects of CAP in the biological environment and on artificial surfaces ${ }^{17,18}$. Cold atmospheric pressure 
argon plasma can inactivate or kill microorganisms by directly reacting with microbial cell membranes or by damaging cell membranes, proteins, and DNA with charged particles and reactive species ${ }^{19}$. A recent randomized controlled trial demonstrated a significant reduction in bacterial load in chronic infected wounds after $5 \mathrm{~min}$ treatment with cold atmospheric argon plasma in vivo ${ }^{19}$. Further research is needed to determine whether argon- and nitrogen-NTAPP reduce the abundance of $C$. acnes indirectly as a result of sebum reduction or by the direct killing of $C$. acnes.

Currently, oral isotretinoin is the most efficient option for treating hyperseborrhea. However, its clinical use is limited by the risk of teratogenicity, liver dysfunction, inflammatory bowel disease, and possible psychological effects. Our results suggest that argon- and nitrogen-NTAPP pulses inhibit lipid production, ductal and sebaceous cell proliferation, and PPARy transcription in sebaceous glands to prevent hyperseborrhea. In addition, the clinical trial in this study indicates that NTAPP therapy exerts sebosuppressive effects without causing apparent thermal damage in the skin. With these results, argonand nitrogen NTAPP treatment can be a promising alternative energy-based therapy targeting sebaceous glands without inducing obvious thermal tissue damage. Increased sebum production and $C$. acnes proliferation in the pilosebaceous unit are important for acne pathogenesis. Given its effects on sebum excretion and porphyrin levels in oily human skin, argon and nitrogen NTAPP pulses can be potentially applied for the treatment of acne vulgaris. Future clinical trials are needed to assess the safety and effectiveness of varied energies of argon- and nitrogen-NTAPP pulses in patients with acne vulgaris and hyperseborrhea.

\section{Material And Methods}

\section{Subjects}

Fifteen healthy volunteers aged 19-35 years were enrolled in this trial. This study was approved by the institutional review board of the Yonsei University College of Medicine (IRB No. 3-2019-0239). All methods were carried out in accordance with the Declaration of Helsinki and Good Clinical Practice as defined under the Korea Food \& Drug Administration and the International Conference on Harmonisation guidelines. The demographic and baseline characteristics are shown in Table 1. Written informed consent was obtained from all participants prior to inclusion in the study (ClinicalTrials.gov identifier:

NCT04917835, 08/06/2021). Before enrollment, casual sebum levels at the surface of the forehead and on the right and left cheeks were measured using a Sebumeter ${ }^{\circledR}$ (SM815; Courage + Khazaka Electronic $\mathrm{GmbH}$, Cologne, Germany). Skin type was classified based on sebum measurements according to the manufacturer's guidelines as follows: $>220 \mu \mathrm{g} / \mathrm{cm}^{2}$ on forehead and $>180 \mu \mathrm{g} / \mathrm{cm}^{2}$ on cheeks, oily type; $100-220 \mu \mathrm{g} / \mathrm{cm}^{2}$ on forehead and $70-180 \mu \mathrm{g} / \mathrm{cm}^{2}$ on cheeks, normal type; $<100 \mu \mathrm{g} / \mathrm{cm}^{2}$ on forehead and $<70 \mu \mathrm{g} / \mathrm{cm}^{2}$ on cheeks, dry type. Only participants with oily skin type were enrolled in the study. At baseline, the mean ( \pm standard deviation [SD]) casual sebum levels on the forehead and both cheeks were $238.2 \pm 58.18$ and $191.7 \pm 51.66 \mathrm{mg} / \mathrm{cm}^{2}$, respectively. All participants had Fitzpatrick's skin phototype III and mild acne vulgaris (grades $1-2$ using the Korean acne grading system) ${ }^{20}$. Volunteers 
were excluded from the study if they had a history of surgical or cosmetic procedures or who had taken systemic retinoids within the prior 6 months; had been treated with topical retinoids within the prior 4 weeks; had acne of grade 3 or higher; had a concurrent systemic disease; had a history of hypertrophic scars or keloids; or were pregnant. To avoid diurnal variation, sebum levels were measured between and 10:00 and 12:00 h. Participants were instructed not to wear cosmetics for $2 \mathrm{~h}$ before the measurements. All measurements were made by the same trained physician in the same room under conditions of 20$22^{\circ} \mathrm{C}$ and $20-40 \%$ humidity conditions after 30 min acclimatization.

\section{Generation of argon-and nitrogen-NTAPP pulses and clinical treatment}

An NTAPP generator (PlaDuo ${ }^{\mathrm{TM}}$; Shenb Co., Ltd., Seoul, Korea) was utilized to generate argon- and nitrogen-NTAPP pulses from inert gaseous sources. Each unexcited gaseous source was loaded at the distal end of the handpiece, and then $2.45 \mathrm{GHz}$ microwave energy converted the loaded sources to plasma in the nozzle. The treatment settings in terms of loading volume and pulse duration were determined according to energy settings; a loading volume of $0.12-0.67 \mathrm{~mL} /$ pulse and a pulse duration of $4-18 \mathrm{~ms}$ at an energy of $0.12-0.75 \mathrm{~J} /$ pulse for argon plasma, and $0.2-1.42 \mathrm{~mL} /$ pulse and $5-36 \mathrm{~ms}$ at $0.5-4 \mathrm{~J} /$ pulse for nitrogen gas.

As approved by the ethics committee, participants underwent three treatment sessions at 1-week intervals. Two passes of argon-NTAPP treatment and two passes of nitrogen-NTAPP treatment were sequentially performed in each session. The forehead, nose, and both cheek areas were treated with two passes of argon- and nitrogen-NTAPP pulses delivered at a pulse energy of $0.8 \mathrm{~J} /$ pulse with a $12 \mathrm{~ms}$ of pulse duration and at a pulse energy of $0.75 \mathrm{~J} /$ pulse delivered with a $7 \mathrm{~ms}$ of pulse duration, respectively, without pretreatment with topical anesthetic cream. The nozzle diameter was $5 \mathrm{~mm}$, and the distance from the nozzle tip to the skin was $10 \mathrm{~mm}$. The treated areas were cooled with icepacks immediately after treatment.

All participants were followed up 2, 4, and 8 weeks after the last session of NTAPP treatment. To investigate adverse events related to plasma treatment, patient symptoms were assessed by physicians. The extent of erythema, edema, pain, oozing, desquamation, postinflammatory hyperpigmentation, and scarring were scored at each visit by grading on a visual analog scale of 0 to 3 ( 0 : absent; 1 : mild; 2 : moderate; 3: severe).

\section{Measurement of porphyrin}

After face washing and 30 min of acclimatization, UV photography was performed using a Mark-Vuß skin analysis imaging system (PSI Plus, Suwon, Korea) at baseline (week 0 ) and 2 weeks after the third session of plasma treatment (week 2). Sebum and porphyrins emit blue and orange fluorescence from UV light sources, respectively. First, to analyze the amount of sebum in the $U$ zone (cheek area and forehead) and the partial T zone (nose), the ratio of the area occupied by blue fluorescence was calculated. The 
amount of porphyrin was calculated by splitting each RGB channel and then expressed as a percentage of the total sebum amount.

\section{Measurement of facial sebum levels}

Next, the casual sebum level was measured using a Sebumeter ${ }^{\circledR}$ in the forehead (mid-glabella) and the right and left cheeks (over the zygoma at the mid-pupillary line). The rate of sebum excretion, which represents the amount of facial sebum excretion for $60 \mathrm{~min}$, was also investigated. Baseline sebum was measured immediately after washing. The subjects then remained in the same room under a controlled environment for $60 \mathrm{~min}$, after which the second sebum level (at $60 \mathrm{~min}$ ) was obtained. The sebum excretion rate was determined by calculating the difference between sebum levels at baseline and 60 $\min$.

\section{Skin biopsy, hematoxylin and eosin staining, immunohistochemistry, and immunofluorescence staining}

Skin punch biopsies ( $3 \mathrm{~mm}$ ) were obtained from the zygomatic area of two of 15 volunteers who completed the study, and 2 weeks after argon- and nitrogen-NTAPP treatment. Paraffin-embedded tissue sections $(4 \mu \mathrm{m})$ were deparaffinized in xylene, rehydrated in an ethanol series, and incubated in blocking buffer (Novocastra, Newcastle upon Tyne, UK; 3\% hydrogen peroxide) for 20 min at room temperature. Slides were incubated overnight at $4^{\circ} \mathrm{C}$ with a rabbit anti-mouse Ki67 antibody (1:200; Abcam, Cambridge, MA, USA; \#ab15580), a rabbit polyclonal anti-4-Hydroxynonenal (4-HNE) antibody (1:100; Abcam, Cambridge, MA, USA; \#ab46545), and a rabbit polyclonal anti-PPAR gamma antibody (1:100; Abcam, Cambridge, MA, USA; \#ab59256), and then washed three times with phosphate buffered saline (PBS). The slides were incubated with HRP-conjugated anti-rabbit IgG (Dako, Carpinteria, CA, USA; \#K4063) at room temperature for $60 \mathrm{~min}$. To stain for Ki67, the slides were treated with diaminobenzidine for 5-10 min after washing and then washed again. Slides were stained with hematoxylin and eosin. Alexa Fluor 488-conjugated rabbit anti-mouse IgG (Thermo Fisher Scientific) was used as a secondary antibody for 4HNE and PPARD staining. Images were captured using an LSM 780 confocal microscope (Carl Zeiss).

\section{Sebocyte culture and NTAPP treatment}

The immortalized human SZ95 sebaceous gland cell line ${ }^{16}$ was cultured in Dulbecco's modified Eagle medium (DMEM)/F-12 culture medium supplemented with $2 \mathrm{mM}$ GlutaMAX I, $10 \mu \mathrm{g} / \mathrm{mL}$ gentamicin, 50 $\mathrm{ng} / \mathrm{mL}$ human epidermal growth factor, 10\% fetal bovine serum (FBS), and 10 mM 4-(2-hydroxyethyl)-1piperazineethanesulfonic acid (all purchased from Gibco BRL, Rockville, MD, USA) and maintained at $37^{\circ} \mathrm{C}$ and $5 \% \mathrm{CO}_{2}$. The medium was replaced every 2 days. SZ95 sebocytes were seeded into 6-well plates and allowed to reach approximately $90 \%$ confluence overnight. To stimulate lipogenesis, sebocytes were treated with a combination of $2 \times 10^{-8} \mathrm{M}$ testosterone and $10^{-4} \mathrm{M}$ linoleic acid (T/LA) for $48 \mathrm{~h}$. All compounds were diluted in dimethyl sulfoxide (DMSO), and then diluted with culture medium (the final concentration of DMSO was $0.1 \%$ ). Argon- or nitrogen-NTAPP pulses were delivered to sebocytes immediately or $24 \mathrm{~h}$ after treatment with T/LA. The 20 or 60 passes of argon-NTAPP pulse at an energy 
of $0.75 \mathrm{~J}$ and a pulse duration of $11 \mathrm{~ms}$, or 5 or 10 passes of nitrogen-NTAPP pulse at an energy of $1.5 \mathrm{~J}$ and a pulse duration of $13 \mathrm{~ms}$ were administered to each well at a $5 \mathrm{~mm}$ distance. All controls were maintained in culture media at room temperature during the experiment to ensure equivalent treatment conditions.

\section{Assessment of cell viability}

SZ95 sebocytes were seeded into six-well plates at a density of $2 \times 10^{5}$ cells/well and then cultured overnight. Cells were irradiated with 20 or 60 pulses of argon-NTAPP at an energy of $0.75 \mathrm{~J}$, and 5 or 10 pulses of nitrogen-NTAPP at an energy of $1.5 \mathrm{~J}$ and then incubated for $48 \mathrm{~h}$. The cells were then incubated with $5 \mathrm{mg} / \mathrm{mL}$ of MTT reagent (3-(4,5-dimethylthiazol-2-yl)-2,5-diphenyltetrazolium bromide) for $2 \mathrm{~h}$. The MTT solution was removed and then $200 \mu \mathrm{L}$ of DMSO was added to dissolve the formazan crystals. The optical density (OD) was measured at $540 \mathrm{~nm}$ using a spectrophotometer.

\section{Immunocytochemistry}

SZ95 sebocytes were grown on coverslips, fixed in acetone, permeabilized with $0.1 \%$ Triton X-100 (SigmaAldrich, St. Louis, MO, USA) in PBS, and then incubated with a rabbit anti-mouse Ki67 (1:200; Abcam; \#AB15580) antibody for $60 \mathrm{~min}$ at $37^{\circ} \mathrm{C}$ (dilution 1:200). Slides were subsequently incubated with a goat anti-rabbit FITC-conjugated secondary antibody (Santa Cruz Biotechnology, Santa Cruz, CA, USA) (dilution 1:100) for 30 min at room temperature, and nuclei were visualized with propidium iodide (PI) (Vector). The cells were examined using an LSM 780 confocal microscope (Carl Zeiss).

\section{Determination of intracellular lipids}

For the quantitative measurement of sebaceous lipids, SZ95 sebocytes (15,000 cells/well) were cultured in 96-well plates (Greiner Bio One, Frickenhausen, Germany) in quadruplicate and then treated with T/LA with or without plasma pulse treatment. Supernatants were then discarded, and $100 \mu \mathrm{L}$ of $1 \mu \mathrm{g} / \mathrm{mL} \mathrm{Nile}$ red (Sigma-Aldrich) solution was added to each well. The plates were incubated at $37^{\circ} \mathrm{C}$ for 20 min, and the emitted fluorescence was measured using a Molecular Devices FlexStation 384II fluorescence image microplate reader (FLIPR, Molecular Devices, San Francisco, CA, USA). The results are presented as percentages of absolute fluorescence units compared with the controls, at excitation and emission wavelengths of 485 and $565 \mathrm{~nm}$, respectively, for neutral lipids. To detect lipid droplets by microscopy, 4\% PFA-fixed cells were incubated with 4,4-difluoro-1,3,5,7,8-pentamethyl-4-bora-3a,4a-diaza-s-indacene 493/503 fluorescence lipid stain $(10 \mu \mathrm{g} / \mathrm{mL}$ in PBS; BODIPY; Thermo Fisher Scientific, Waltham, MA, USA) for $7 \mathrm{~min}$ at $37^{\circ} \mathrm{C}$. After incubation, the slides were washed with $0.5 \%$ BSA-PBS and imaged under fluorescence microscopy. Images were captured using a LSM 780 confocal microscope (Carl Zeiss).

\section{Real-time quantitative polymerase chain reaction}

RNA was extracted from SZ95 sebocytes using TRIzol reagent (Thermo Fisher Scientific), according to the manufacturer's protocol. RNA was quantified using a NanoDrop 2000c (Thermo Fisher Scientific) and cDNA was synthesized using a cDNA Synthesis kit (Thermo Fisher Scientific). TaqMan real-time polymerase chain reaction (PCR) assays were performed to analyze mRNA levels (Applied Biosystems, 
Foster City, CA, USA). TaqMan probes for PPARy (Hs01115514_m1, Thermo Fisher Scientific) and sterol regulatory element binding protein-1 (SREBP1) (Hs01088678_g1, Thermo Fisher Scientific) were used. The relative expression of each gene was normalized to the mean level of GAPDH mRNA (Hs02786624_g1, Thermo Fisher Scientific).

\section{Statistical analysis}

Statistical analyses were performed using Prism 6 (GraphPad) and R version 3.4.3. All tests were twotailed. An unpaired t-test was used to compare data between the two groups. Statistical significance was set at ${ }^{\star} P<0.05,{ }^{* \star} P<0.01$, with error bars used to indicate the standard error of the mean (SEM).

\section{Declarations}

\section{Acknowledgements}

We thank Sunny Kang (Shenb Co., Ltd., Seoul, Korea), Bora Kim (Shenb Co., Ltd.), and Min Choi (Shenb Co., Ltd.) for their technical support. We also thank Anthony Thomas Milliken, ELS at Editing Synthase (https://editingsynthase.com) for his help editing this manuscript.

\section{Author contributions}

S-E.K. and SE.L. initiated and performed the experiments, SB.C., S-E.K., and SE.L. designed and conducted the research, S-J.L. and DS.Y analyzed the data, SB.C., S-J.L., and SE.L. wrote the paper, and C.C.Z. and SE.L. edited the manuscript. All authors reviewed the manuscript.

Competing interests statement: The authors declare no conflicts of interest.

\section{References}

1. Park, J. et al. Non-Thermal Atmospheric Pressure Plasma Efficiently Promotes the Proliferation of Adipose Tissue-Derived Stem Cells by Activating NO-Response Pathways. Sci Rep, 6, 39298 https://doi.org/10.1038/srep39298 (2016).

2. Gan, L. et al. Medical applications of nonthermal atmospheric pressure plasma in dermatology. J Dtsch Dermatol Ges, 16, 7-13 https://doi.org/10.1111/ddg.13373 (2018).

3. Cheng, K. Y. et al. Wound Healing in Streptozotocin-Induced Diabetic Rats Using AtmosphericPressure Argon Plasma Jet. Sci Rep, 8, 12214 https://doi.org/10.1038/s41598-018-30597-1 (2018).

4. Heinlin, J. et al. Randomized placebo-controlled human pilot study of cold atmospheric argon plasma on skin graft donor sites. Wound Repair Regen, 21, 800-807 https://doi.org/10.1111/wrr.12078 (2013).

5. Kubinova, S. et al. Non-thermal air plasma promotes the healing of acute skin wounds in rats. Sci Rep, 7, 45183 https://doi.org/10.1038/srep45183 (2017). 
6. Liu, J. R., Xu, G. M., Shi, X. M. \& Zhang, G. J. Low temperature plasma promoting fibroblast proliferation by activating the NF-KB pathway and increasing cyclinD1 expression. Sci Rep, 7, 11698 https://doi.org/10.1038/s41598-017-12043-w (2017).

7. Weyandt, G. H., Benoit, S., Becker, J. C., Bröcker, E. B. \& Hamm, H. [Controlled layered removal of anogenital warts by argon-plasma coagulation]. J Dtsch Dermatol Ges, 3, 271-275 https://doi.org/10.1111/j.1610-0387.2005.04757.x (2005).

8. Wiegand, C. et al. Antimicrobial impact of cold atmospheric pressure plasma on medical critical yeasts and bacteria cultures. Skin Pharmacol Physiol, 27, 25-35 https://doi.org/10.1159/000351353 (2014).

9. Elsaie, M. L. \& Kammer, J. N. Evaluation of plasma skin regeneration technology for cutaneous remodeling. J Cosmet Dermatol, 7, 309-311 https://doi.org/10.1111/j.1473-2165.2008.00411.x (2008).

10. Fitzpatrick, R. et al. A histopathologic evaluation of the Plasma Skin Regeneration System (PSR) versus a standard carbon dioxide resurfacing laser in an animal model. Lasers Surg Med, 40, 93-99 https://doi.org/10.1002/lsm.20547 (2008).

11. Kilmer, S., Semchyshyn, N., Shah, G. \& Fitzpatrick, R. A pilot study on the use of a plasma skin regeneration device (Portrait PSR3) in full facial rejuvenation procedures. Lasers Med Sci, 22, 101109 https://doi.org/10.1007/s10103-006-0431-9 (2007).

12. Kim, H., Kim, H. J., Kim, H. K., Hong, J. Y. \& Cho, S. B. Effects of argon and nitrogen plasma pulses on the skin and skin appendages in an in vivo animal model. Skin Res Technol, 26, 81-90 https://doi.org/10.1111/srt.12767 (2020).

13. Cornelius, C. E. 3, Ludwig, G. D. \& rd \& Red fluorescence of comedones: production of porphyrins by Corynebacterium acnes. The Journal of investigative dermatology, 49, 368-370 (1967).

14. McGinley, K. J., Webster, G. F. \& Leyden, J. J. Facial follicular porphyrin fluorescence: correlation with age and density of Propionibacterium acnes. The British journal of dermatology, 102, 437-441 https://doi.org/10.1111/j.1365-2133.1980.tb06557.x (1980).

15. Bauer, G., Sersenová, D., Graves, D. B. \& Machala, Z. Cold Atmospheric Plasma and Plasma-Activated Medium Trigger RONS-Based Tumor Cell Apoptosis. Sci Rep, 9, 14210 https://doi.org/10.1038/s41598-019-50291-0 (2019).

16. Zouboulis, C. C., Seltmann, H., Neitzel, H. \& Orfanos, C. E. Establishment and characterization of an immortalized human sebaceous gland cell line (SZ95). The Journal of investigative dermatology, 113, 1011-1020 https://doi.org/10.1046/j.1523-1747.1999.00771.x (1999).

17. Bourke, P., Ziuzina, D., Han, L., Cullen, P. J. \& Gilmore, B. F. Microbiological interactions with cold plasma. J App/ Microbiol, 123, 308-324 https://doi.org/10.1111/jam.13429 (2017).

18. Daeschlein, G. et al. In vitro susceptibility of multidrug resistant skin and wound pathogens against low temperature atmospheric pressure plasma jet (APPJ) and dielectric barrier discharge plasma (DBD). Plasma Processes and Polymers, 11, 175-183 (2014). 
19. Isbary, G. et al. Successful and safe use of 2 min cold atmospheric argon plasma in chronic wounds: results of a randomized controlled trial. The British journal of dermatology, 167, 404-410 https://doi.org/10.1111/j.1365-2133.2012.10923.x (2012).

20. Sung, K. J. et al. Korean acne grading system. Korean Journal of Dermatology, 42, 1241-1247 (2004).

\section{Figures}

A

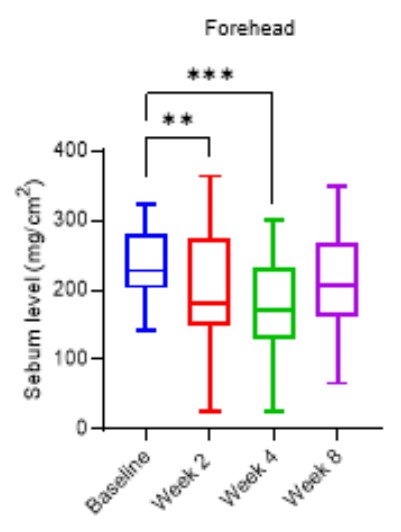

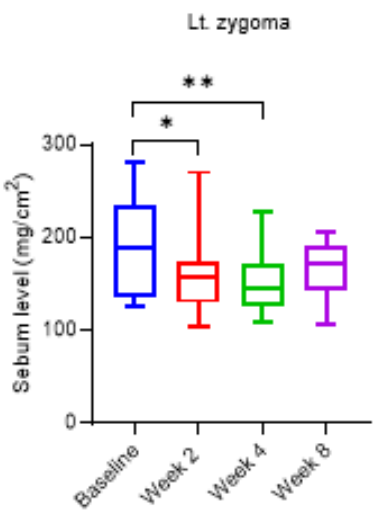

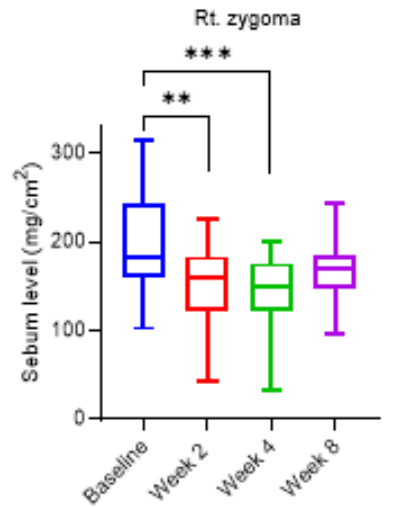

B

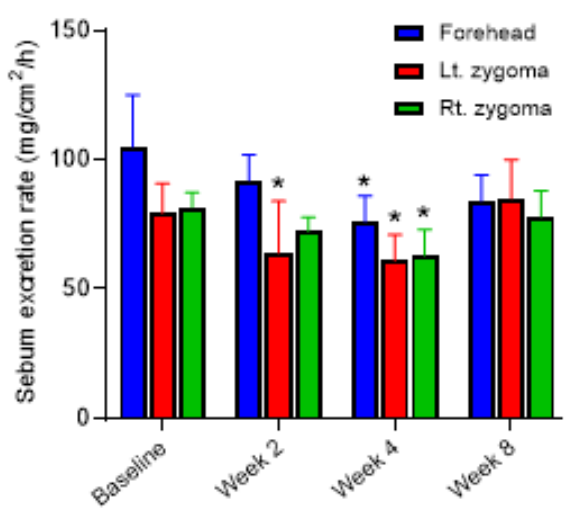

Figure 1

Effects of argon- and nitrogen-NTAPP pulses on facial sebum excretion in subjects with oily skin. Casual sebum level $(A)$ and sebum excretion rate $(B)$ were measured using a sebumeter at baseline and at follow-up visits on weeks 2,4 , and 8 . Values are presented as the mean \pm standard error of the mean $(\mathrm{SEM} ; \mathrm{n}=15)$. Data were analyzed by Wilcoxon matched-pairs signed rank test $\left({ }^{\star} \mathrm{P}<0.05,{ }^{\star \star} \mathrm{P}<0.01\right.$, ${ }^{\star \star \star} \mathrm{P}$ $<0.001$, vs. baseline). 
A
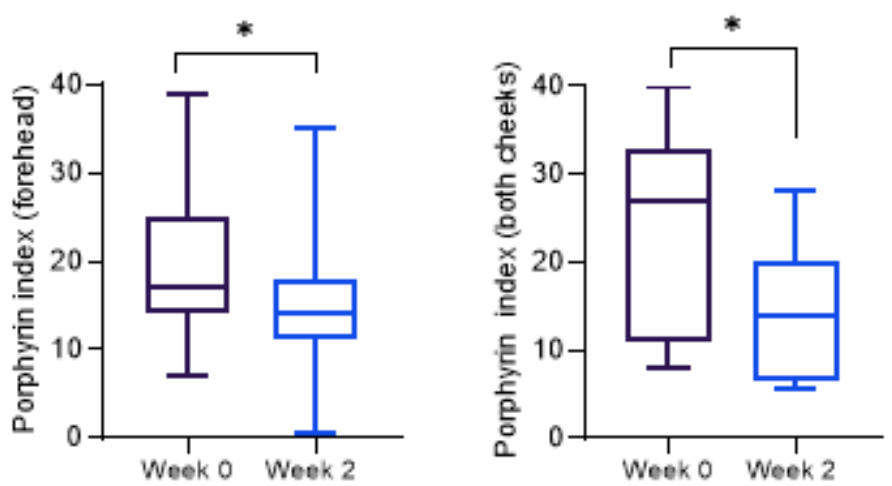

B

Baseline

Week 2
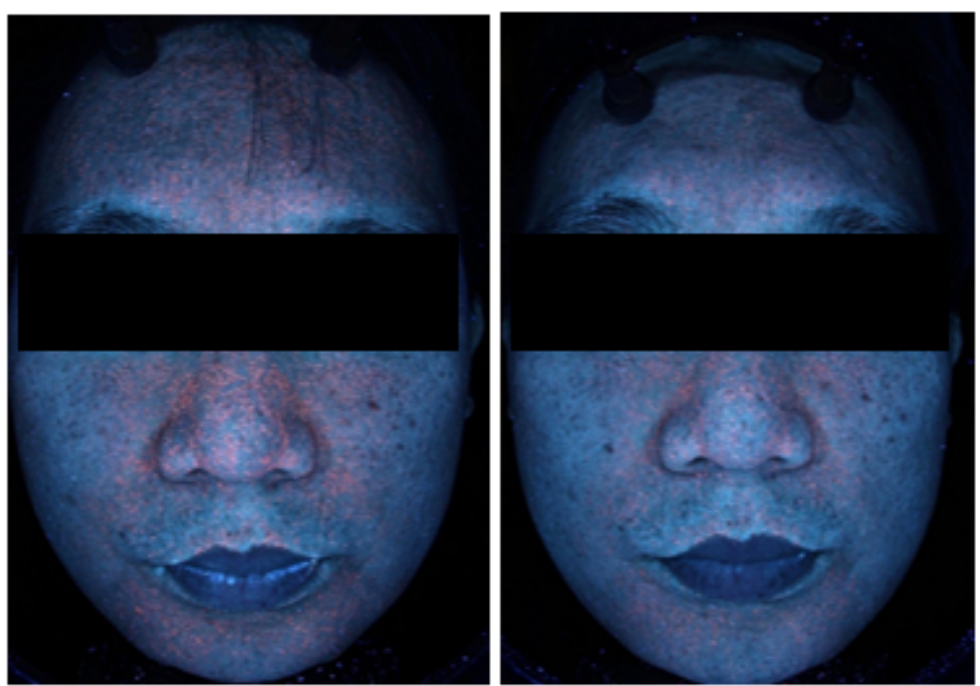

\section{Figure 2}

Argon- and nitrogen-NTAPP pulses decreased porphyrins produced by C. acnes in seborrheic areas of oily human skin. (A) Porphyrin index determined by a digital photography analyzer of ultraviolet photographs taken at baseline and 2 weeks after argon- and nitrogen-NTAPP pulses treatment. Values represent the mean \pm SEM $(n=7)$. Data were analyzed Wilcoxon matched-pairs signed rank test ( ${ }^{*} P<0.05$ vs. baseline). (B) Representative ultraviolet photographs at baseline and week 2. 
Subject 1

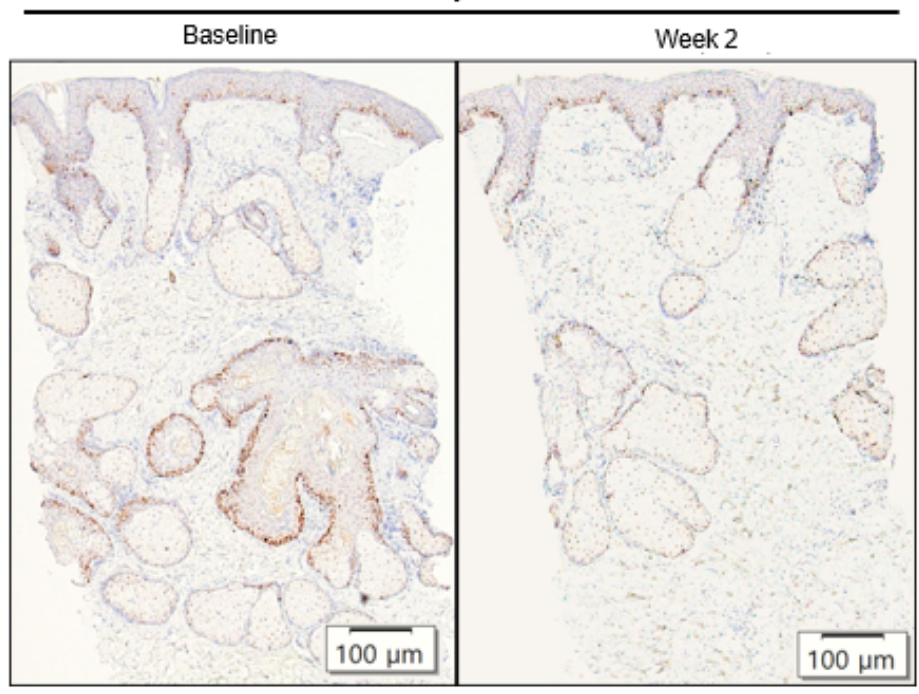

Subject 2

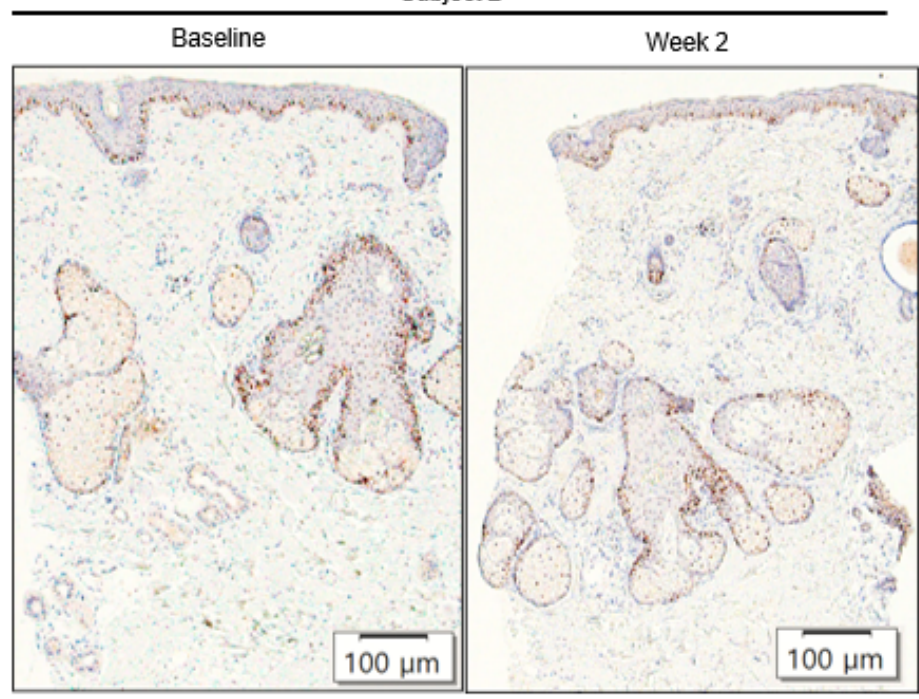

\section{Figure 3}

Representative immunohistochemistry of Ki-67 in facial human skin at baseline and 2 weeks after plasma treatment. Positively immunostained cells appear brown, and nuclei are blue. Scale bars: $100 \mu \mathrm{m}$. 

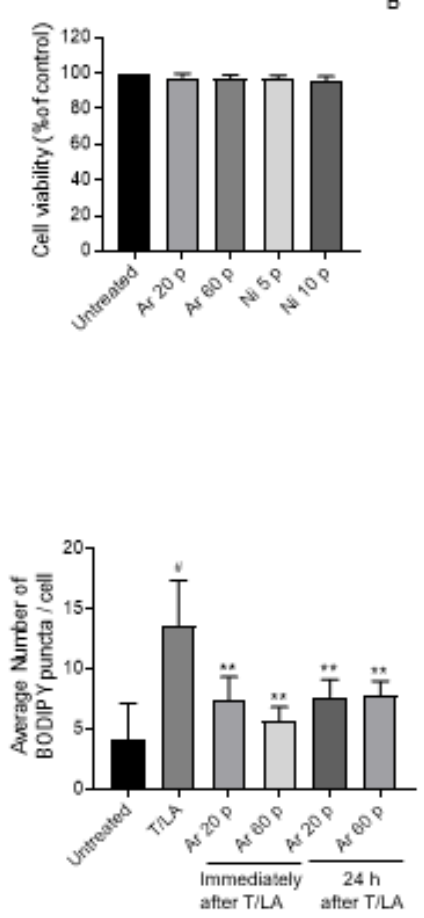

E

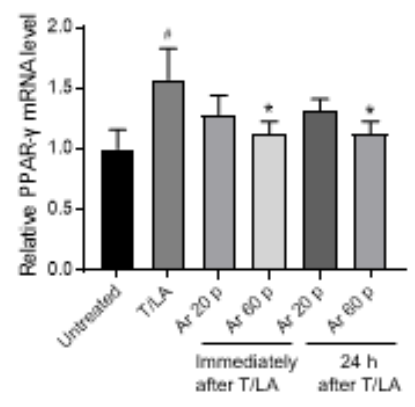

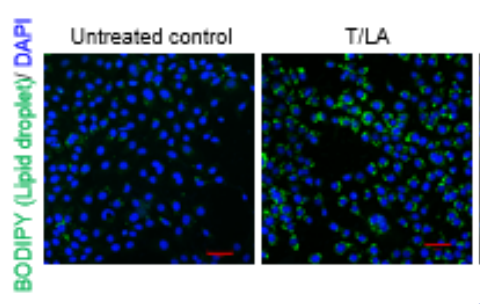

Immediately after T/LA treatment

Ar, $0.75 \mathrm{~J}, 20$ pulses Ar, $0.75 \mathrm{~J}, 60$ pulses $A r, 0.75 \mathrm{~J}, 20$ pulses $A r, 0.75 \mathrm{~J}, 60$ pulses

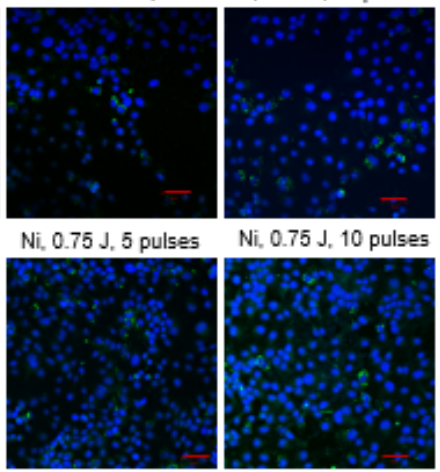

D
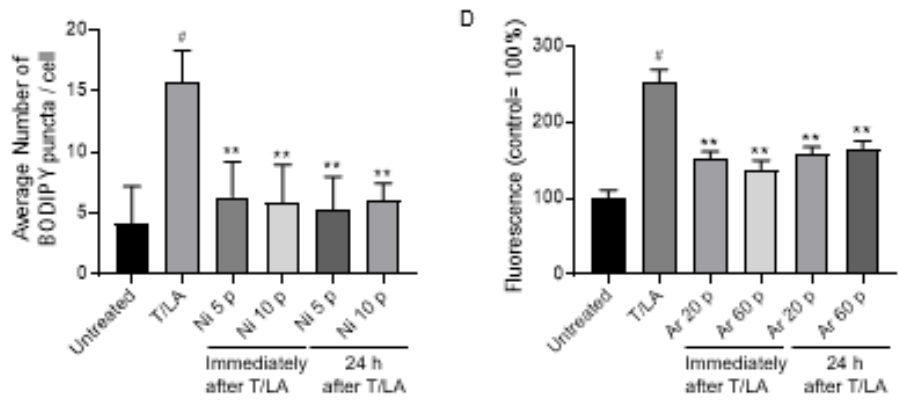

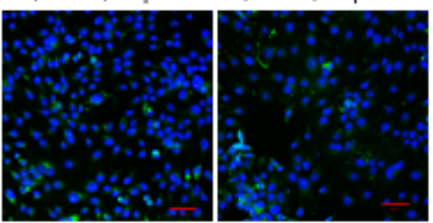

$\mathrm{Ni}, 0.75 \mathrm{~J}, 5$ pulses

Ni, $0.75 \mathrm{~J}, 10$ pulses
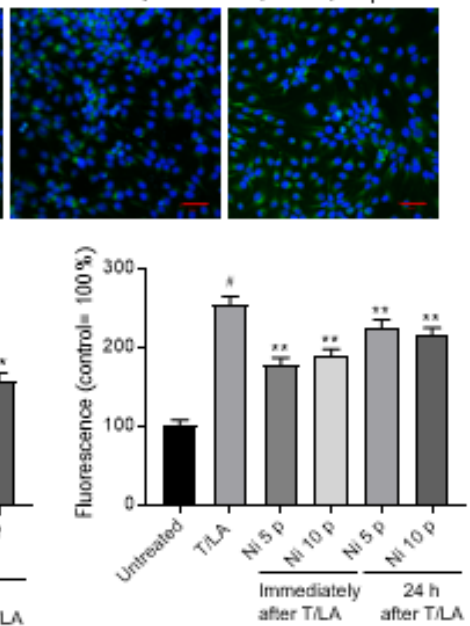

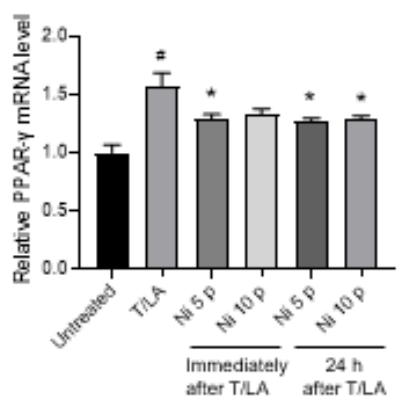

\section{Figure 4}

Effect of argon- and nitrogen-NTAPP pulses on the viability and lipid synthesis of human sebocytes. (A) SZ95 sebocytes were treated with argon-and nitrogen-NTAPP pulses for $48 \mathrm{~h}$, and then subjected to an MTT assay. Data are shown as the mean \pm SEM $(n=3)$. (B) Representative confocal immunofluorescence images of BODIPY in SZ95 sebocytes treated with T/LA alone for $48 \mathrm{~h}$ or in combination with argon-and nitrogen-NTAPP pulses immediately or $24 \mathrm{~h}$ after T/LA treatment. Scale bars represent $50 \mu \mathrm{m}$. (C) Average numbers of BODIPY-positive puncta in SZ95 sebocytes are shown as a bar graph. Data indicate the mean \pm SEM. $(n=25)$. (D) Neutral lipids were quantified by Nile Red staining and then measured using a fluorometric imaging plate reader. Data indicate the mean \pm SEM. (E) PPAR\ mRNA expression was analyzed by quantitative PCR analysis. Results are normalized to the internal control GAPDH and are shown relative to those in control cells incubated in culture medium. Data are expressed as the mean \pm SEM of three independent experiments. $\# P<0.05$ versus untreated control and ${ }^{*} P<0.05,{ }^{\star} * P<0.01$ versus T/LA-treated cells by two-tailed unpaired Student's t test. T/LA, testosterone/linoleic acid; Ar, argon; Ni, nitrogen; $p$, pulses. 

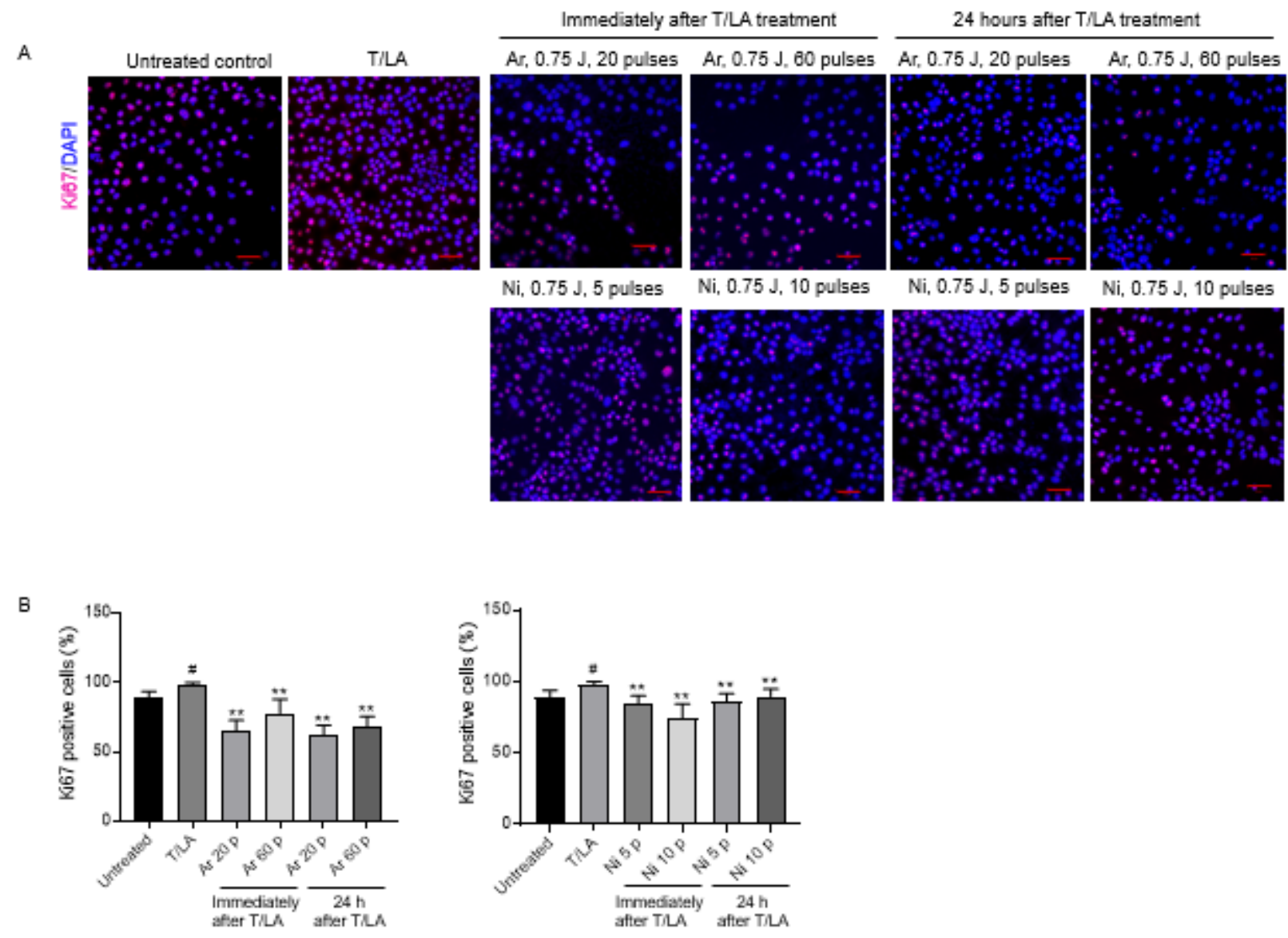

\section{Figure 5}

Argon- and nitrogen-NTAPP pulses regulate the proliferation of cultured SZ95 sebocytes. (A) Immunofluorescence of SZ95 sebocytes treated with T/LA alone for $48 \mathrm{~h}$ or in combination with argonand nitrogen-NTAPP pulses stained for Ki67. The scale bars represent $50 \mu \mathrm{m}$. (B) The percentage of nuclear Ki-67 positive cells to the total DAPI positive cells in T/LA-treated SZ95 cells with or without cotreatment with argon-and nitrogen-plasma pulses. Data are shown as the mean $\pm \mathrm{SEM}$. \#P $<0.05$ versus untreated control and ${ }^{* *} \mathrm{P}<0.01$ versus T/LA-treated cells by two-tailed unpaired Student's $t$ test. T/LA, testosterone/linoleic acid; $\mathrm{Ar}$, argon; $\mathrm{Ni}$, nitrogen; $\mathrm{p}$, pulses. 